\title{
ANALYSIS OF EXPRESSION AND POSTTRANSLATIONAL MODIFICATION OF AN UNCHARACTERIZED ISOFORM OF THE TRANSLATION FACTOR EIF5A
}

\author{
Patrícia T. Shinta (IC), Karina D. Pereira (PG), Augusto D. Luchessi (PQ), Rogerio F. Lourenço (PQ).
}

\begin{abstract}
The translation factor elF5A is a protein containing the amino acid residue hypusine, which has never been found in other proteins. In human cells, an EIF5A1 transcript variant referred to as variant $A$ is expected to encode the elF5A1 isoform $A$, which has an additional $\mathrm{N}$-terminal extension with respect to the canonical isoform $B$. Here, we validated the isoform $A$ in HeLa cells and showed that variant $A$ is able to produce both elF5A1 isoforms. Furthermore, isoform A was proved to undergo acetylation at least when it is exogenously expressed.
\end{abstract}

Key words: elF5A, transcript variant, protein isoform

\section{Introduction}

The translation factor elF5A is a highly conserved protein in eukaryotes and archaea, and it undergoes an essential and currently particular posttranslational modification called hypusination ${ }^{1}$. Two gene loci corresponding to elF5A (EIF5A1 and EIF5A2) are present in the genome of human cells. While EIF5A2 seems to be relevant in tumorigenisis, EIF5A1 is critical for several important cellular processes, including protein synthesis, mRNA transport and degradation, stress granule assembly and apoptosis ${ }^{1}$. Five EIF5A1 transcript variants are currently known (A, $\mathrm{B}, \mathrm{C}, \mathrm{D}$ e X5). Variants $\mathrm{B}, \mathrm{C}, \mathrm{D}$ and $\mathrm{X} 5$ code for the canonical elF5A1 isoform (referred to as isoform $B$ ), whereas variant $A$ appears to be necessary for expression of a different isoform, which is characterized by an additional 30 amino acid residues $\mathrm{N}$-terminal extension (isoform $\mathrm{A}$ ). To date all efforts were concentrated on the isoform $B$, and virtually no information is available to provide any clue concerning the function played by the isoform A. In this work, we analyzed expression and posttranslational modification of this uncharacterized elF5A1 isoform.

\section{Results and Discussion}

Western blotting analyses using antibodies against elF5A1 showed that HeLa cells produce both isoform $A$ and isoform $B$. With the aim of expressing elF5A1 isoforms fused to the FLAG tag, plasmids were constructed by reverse transcription of variant $A$ and variant $B$. Surprisingly, elF5A1 isoforms $A$ and $B$ were detected in extracts from HeLa cells transfected with the plasmid obtained from variant $A$, indicating that this transcript is responsible for the synthesis of both isoforms. As expected, only isoform B was identified in cells transfected with the construction corresponding to variant $B$. Extracts from cells overexpressing both FLAGtagged isoforms were used in immunoprecipitation experiments with an anti-FLAG antibody. After resolving the resulting samples in SDS-PAGE, the bands were isolated and analyzed by mass spectrometry. Only peptides common to both isoforms were identified in this analysis. For both isoforms, proper acetylation at equivalent residues (Lys ${ }^{77}$ for isoform A and Lys ${ }^{47}$ for isoform B) were identified, but no other posttranslational modification was identified. Taking into account that hypusination can impair cleavage after the resulting modified lysine, an additional protein search allowing for up to two miscleavages was carried out. However, the hypusine residue was not identified in the corresponding peptide of any isoform, suggesting that this posttranslational modification is at least compromised in exogenously expressed proteins.

\section{Conclusions}

In this work, the existence of the human elF5A1 isoform $A$ in HeLa cells and its production from the transcript variant $A$ were validated. Additionally, the same transcript was shown to be involved in the production of isoform B. Although no data regarding to the hypusination status of isoform A has been obtained, it was demonstrated that this protein isoform is target of acetylation, what is also the case of isoform $B$.

\section{Acknowledgement}

We are grateful to Mass Spectrometry Laboratory (CNPEM). This work was supported by grants of FAEPEX- Unicamp, CNPq and FAPESP.

\footnotetext{
${ }^{1}$ Rossi D, Kuroshu R, Zanelli CF, Valentini SR. eIF5A and EF-P: two unique translation factors are now traveling the same. Wires RNA. 2014. 5: 209-222.
} 